\title{
Overview of a Supersonic Probe for Solid Propellant Rocket CCP Collection
}

\author{
S. Carlotti ${ }^{1}$, F. Maggi ${ }^{2}$, R. Bisin ${ }^{3}$, S. Dossi ${ }^{4}$, L. Galfetti ${ }^{5}$ \\ Politecnico di Milano, Department of Aerospace Science and Technology, 34, via LaMasa, 20156, Milan, Italy \\ D. Saile ${ }^{6}$, A. Gühlan ${ }^{7}$ \\ German Aerospace Center DLR, Supersonic and Hypersonic Technology Department, Linder Höhe, 51147, \\ Cologne, Germany \\ C.Groll $^{8}$, T. Langener ${ }^{9}$ \\ European Space Agency (ESA/ESTEC), Keplerlaan 1, 2201 AZ Noordwijk, Netherlands
}

The present work aims at giving a comprehensive overview of the current development status of an intrusive probe, capable of collecting the condensed combustion products present in the exhaust of a solid rocket motors. The innovative technique was conceived in the EMAP (Experimental Modelling of Alumina Particulate in Solid Booster) framework, a project aiming at characterizing the alumina in terms of size, temperature and spatial distribution to gain detailed information for climatological impact assessment. A supersonic probe was sized to handle a progressive deceleration and cooling of the exhaust gas, as well as the quenching and collection of the suspended particles in a pressure-controlled chamber. The task was achieved by through a quasi 1D gas dynamics code based on the Shapiro method and the normal shock wave theory, which was verified against a hybrid $2 \mathrm{D}$ axial-symmetric mesh whose turbulent flow field was solved using the DLR-TAU CFD code. The robustness of the system has been investigated performing a sensitivity and an uncertainty analysis, exploring uncertainties propagation through the numerical code based on Shapiro equations. The sensitivity analysis enabled to define a ranking of importance for the uncertainties on the probe behavior; the uncertainty analysis allowed to estimate failures of the system and/or of the code. Cold flow tests carried out at the vertical facility of DLR-Cologne enabled to gain a proof of concept for both fluid dynamic behavior and collection methodology.

\section{Nomenclature}

D diameter

f Friction factor

K Specific heat ratio

I Elementary effects indexes

L Length

L* Mixing Length

M Mach number

$\mu^{*} \quad$ Absolute mean

$\mathrm{P} \quad$ Pressure

$P_{t} \quad$ Collection chamber pressure

${ }^{1}$ PhD Student, Dept. Aerospace Science and Technology, Via La Masa 34.

${ }^{2}$ Assistant Professor, Dept. Aerospace Science and Technology, Via La Masa 34, AIAA Senior Member.

${ }^{3}$ PhD Student, Dept. Aerospace Science and Technology, Via La Masa 34.

${ }^{4}$ Post-doctoral Research Fellow, Dept. Aerospace Science and Technology, Via La Masa 34.

${ }^{5}$ Full Professor, Dept. Aerospace Science and Technology, Via La Masa 34.

${ }^{6} \mathrm{PhD}$ Student, Supersonic and Hypersonic Technology Department, Linder Höhe, 51147.

${ }^{7}$ Head of Supersonic and Hypersonic Technology Department, Linder Höhe, 51147.

${ }^{8}$ Intern, European Space Agency (ESA/ESTEC), Keplerlaan 1, 2201 AZ Noordwijk.

${ }^{9}$ Senior, European Space Agency (ESA/ESTEC), Keplerlaan 1, 2201 AZ Noordwijk. 


$\begin{array}{ll}\mathrm{Re} & \text { Reynolds number } \\ \sigma & \text { Standard deviation } \\ \mathrm{T} & \text { Temperature } \\ T_{f} & \text { Collection chamber temperature } \\ X_{e v} & \text { Evaporation rate } \\ x_{s w} & \text { Shock wave }\end{array}$

\section{Introduction}

$\mathrm{T}_{\mathrm{s}}^{\mathrm{H}}$ HE assessment about the environmental impact of human space access activities requires precise determination of chemical, physical, and radiative interaction between the plume dumped from a rocket launcher and the atmosphere. Such emissions are composed by the products of propellant combustion, mainly containing alumina particles, hydrogen chloride, and carbon monoxide. Latest studies have shown that alumina particles, i.e., oxidized aluminum which is present in the solid rocket propellant to improve the performances, may have a significant ozone depletion effect [1]. If the attention is focused on the particulate evolution from the rocket core flow to the final atmospheric discharge, modeling and experimental knowledge is limited to some sets of databases collected by Hermsen in the 80s [2] and by Sambamurthi in the 90s [3]. Computer modelling used to monitor the plume development and the corresponding environmental evolution after a launch, i.e., computational fluid dynamics, feature large uncertainties in the state, size, and distribution of alumina at the inlet boundary of the domain. The lack of detailed information can lead to inaccuracies in the prediction of plume conditions and its short- and long-term climatological impact.

Within the frame of the Clean Space ESA initiative, a project concluded in 2015 and called ATILA highlighted the importance of aerosols in the depletion of atmospheric ozone [4]. Several papers can be found in support to these conclusions in the open literature. This role was attributed to the condensation of chlorine and moisture on solid particulate suspended in the atmosphere, e.g. on ice suspensions over the polar regions or on alumina powder contained in volcano ashes, see Refs [5-7]. For the aforementioned reasons, a detailed analysis of plume effect on atmosphere requires the knowledge of the distribution of the alumina particulate ejected from rocket nozzles, including mean size, span, distribution, particle morphology, chemical composition, etc.

The EMAP (Experimental Modelling of Alumina Particulate in Solid Booster) activity is a project funded under ESA's Basic Technology Research Program (TRP) for the measurement technique improvement and characterization of the plume exhausted from a sub-scale metalized solid rocket motor (SRM) resembling the exhaust conditions of Ariane 6 booster. The project is an international cooperative action leaded by the German Aerospace Center-Cologne (DLR, Germany) and involving the Swedish Defence Research Agency (FOI, Sweden) and the Space Propulsion Laboratory of Politecnico di Milano (SPLab-POLIMI, Italy). The project aims at characterizing the alumina in terms of size and spatial distribution, change in phase-state, temperature, and velocity. In the present framework, the SPLabPOLIMI was in charge of developing a probe to be placed close to the nozzle exit of a solid rocket motor, capable of collecting the condensed combustion products present in the supersonic exhaust.

The present work aims at giving a comprehensive overview on the current state of the supersonic probe development, spanning from the detailed design to the fluid dynamic characterization and the off-design analysis.

\section{Literature Survey}

Metal agglomerates lifetime can be roughly divided into different steps:

- the incipient agglomeration at the release of the surface;

- the injection from the burning surface and consequent behavior inside the core flow;

- the evolution inside the convergent-divergent nozzle.

The release of liquid droplets from the burning surface in a cross-flow environment can generate breakup immediately after emission, easing metal combustion. Rather, the processes acting on liquid particles in the nozzle range from coalescence, due to reciprocal impacts, to breakup, due to high velocity gradient possibly leading to the critical Weber number. Hence, condensed combustion products (CCPs) collection during rocket tests at nozzle exit section is essential to provide information about alumina to monitor the plume development and the corresponding environmental evolution after a launch. Great effort was put in the past on the development of impact collectors. These devices are based on the impact of the particles trailed by a supersonic flow on a collection plate. Microscopy observations would be performed on the particles collided with the collection plate in order to have an insight into the CCPs particle size and structure [8] [9]. The technique was not optimal since it did not take into account the possible 
effect of shock waves and coalescence in the exhaust plume, these last processes leading to liquid particle shattering and coalescence, respectively. These studies pointed out that the CCP particle size was slightly affected by the combustion chamber pressure (i.e., low pressure promotes smaller particles [9]), the propellant composition, the expansion ratios, the residence time, and, the initial metal particle diameter. The initial metal particles diameter inside the motor was one order magnitude lower with respect to the CCP size, clearly suggesting that the coalescence process occurred in the nozzle [8]. Sehgal [10] constructed a completely enclosed stainless steel tank in which the motor was fired. The exhaust products condensed on the wall of the tank and were swept from it with a soft nylon brush. The method enables to obtain the detailed size distribution averaged over the duration of firing, for the price of an extreme cumbersome system not applicable to a real firing test. However, it is completely complementary with the spectrophotometric approach by Dobbins [11]. Spectrophotometric tests were conducted using transmission measurements at three wavelengths of light. It measured a well-defined mean diameter of a large number of particles leaving the rocket nozzle. In [12], Carns presented a device for scrubbing exhaust gases generated during hot fire tests. The apparatus targeted the reduction of pollutants dumped in the atmosphere, without compromising rocket performance. The scrubber was designed to deal with pollutants including gaseous hydrochloric acid, aluminum oxide, and, lead. Although the gas scrubber by Carns has the capability of managing the full exhaust plume amount, the remarkable size of the device might be a problem in practical applications, and the particles are separated by the gas thanks to quenching liquid and filtering not conceived to maintain particles properties. Several techniques for trapping the exhaust products in the vicinity of the nozzle exit have been developed. Kessel [13] suggested a supersonic probe with a filter screen port for particles collection. The probe is prone to localized effect, ingesting a limiting amount of mass and, as in the case of Carns [12], the filtering may lead to variations in the particles properties

\section{Probe concept}

The operational version of the probe is made by two main parts [14], merging the concept of a supersonic collection methodology [13] and a scrubber segregating the particles from the gas with a quenching liquid sprayed by sprinklers [12]. The logical scheme of the probe is shown in Fig. 1. The flow coming from the external supersonic rocket nozzle is captured by a straight inlet duct, drilled in a high-temperature-resistant graphite tip. After that, a secondary gas, nitrogen, is radially injected enabling a progressive deceleration and cooling of the primary flow in such a way that any strong shock is delayed, avoiding excessive temperature jump and particles break up. After a straight channel, which ensures the time and space for complete mixing, the flow enters into a conical divergent channel where the supersonic-to-subsonic transition occurs by means of a shock wave. The position of the shock is defined by the global design which ensures a passive control of the downstream pressure.

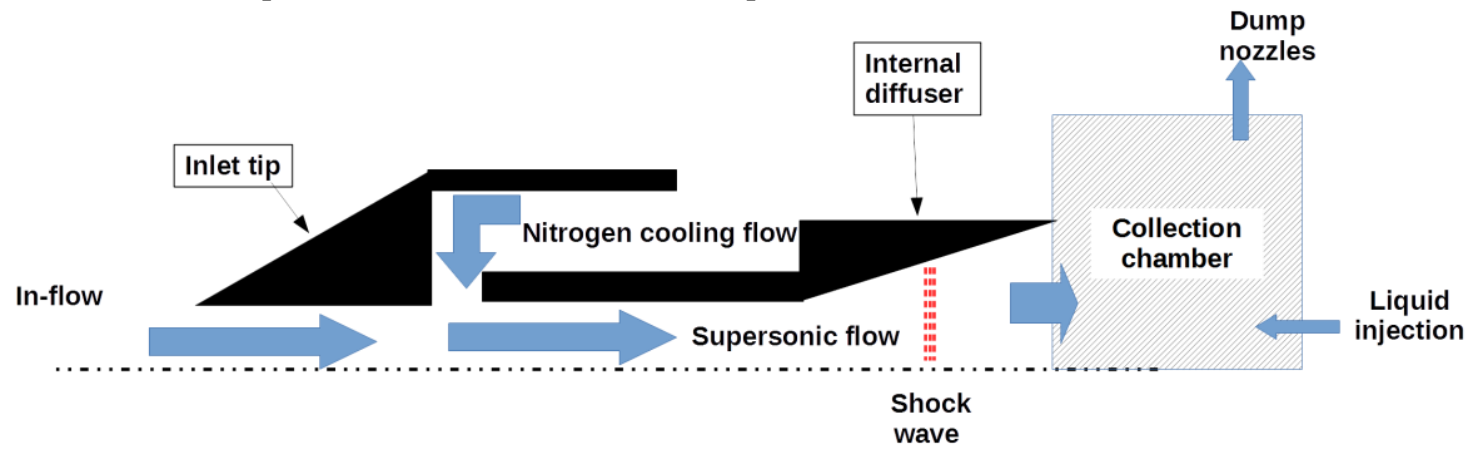

Fig. 1 Logical scheme of the SPLab Supersonic Probe.

Table 1. Relevant probe dimensions

\begin{tabular}{l|cc} 
& Length L, $[\mathrm{cm}]$ & Diameter D, $[\mathrm{cm}]$ \\
\hline Inlet Duct & 3.5 & 0.5 \\
Mixingh Chamber & 8.0 & 1.3 \\
Divergent Duct & 7.2 & 3.7 \\
\hline
\end{tabular}

Once the gas carrier enters the collection chamber, the not-reacting quenching liquid is sprayed in counter-flow and collects the condensed combustion products in a pressure-controlled tank, where a set of calibrated exhaust nozzles allows to control the flow-rate to the ambient. 
In detail, the main dimensions are listed in Table 1, while the main features of the conceived probe are hereby listed:

- the collected particles will be representative of the exhausted plume content, since the probe will be placed close to the exit section of the nozzle, avoiding particles mixing with the external air and post-combustion effects. The probe is able to ingest approximately $4.4 \mathrm{~g} / \mathrm{s}$;

- the operational time will be of 0.5 to 1 second, after which the probe design enables a fast reconditioning and particles post collection analysis;

- chemical and physical alterations of the particles have been minimized. As far as the former is concerned, nitrogen has been selected as secondary gas, due to its inert nature and its use in some quench bombs [15] [16], while tetrachloroethylene will serve as quenching liquid since it is more stable with respect to other solutions and due to the consolidated experience of the SPLab team in its usage in quench bombs. As far as the physical alteration is concerned, any strong shock wave inside the probe is delayed until the particles are in a solid state;

- the supersonic-to subsonic transition is set after the complete cooling of the primary flow in a conical divergent. Strong shock wave inside the straight channel (i.e., the mixing duct) has been avoided due to fluid dynamic instabilities that could result in a probe failure (i.e., bow shock formation in front of the probe);

- a passive thermal management will be applied during the hot flow tests: high temperature thermal protections and a movable and non-toxic shield between the exhaust and the probe will grant the correct exposure and protection of the probe.

\section{Fluid Dynamic Analysis}

The Quasi-1D gas dynamics was employed to support the feasibility and the off-design study of this system. A numerical model based on the Shapiro method and normal shock wave theory was built (POLIRocket-V2) [17]. The software is based on a set of quasi-1D, steady-state, compressible gas dynamic equations, capable of accounting for different phenomena such as area variation, wall friction, heat exchange, chemical reactions, change of phase, mixing of gases which are injected into the main stream, as well as changes in molecular weight and specific heat.

\section{Table 2. Theoretical exhaust conditions.}

\begin{tabular}{l|c}
\hline Mach number M, [-] & 3.36 \\
Static temperature Tin, $[\mathrm{K}]$ & 2227 \\
Static pressure P, $[$ bar $]$ & 0.53 \\
Specific heat ratio k, $[-]$ & 1.16 \\
Gas constant R $[\mathrm{J} / \mathrm{kg} \mathrm{K}]$ & 298 \\
\hline
\end{tabular}

The main drawbacks of this approach are a critical sensitivity to $M=1$ causing numerical instability and an underlying continuity requirement for variables. The boundary conditions and the main features for each component are the following:

- the inlet duct is an adiabatic constant cross-sectional channel with friction effects evaluated from the wellknown Blasius equation, with a nominal expected Re of $17 \times 10^{3}$ :

$$
f=\frac{0.0791}{R e^{0.25}}, \text { for } 2.1 \times 10^{3}<R e<10^{5}
$$

- the supersonic mixing in the straight channel is modeled with a progressive secondary flow addition (uniform differential mass flow rate) with a local recomputation of average properties of the fluid. Since according to Kessel [9], the merging of different boundary layers or stagnant presence of the cooling inert gas inside the mixing straight duct could lead to possible fluid dynamic instabilities, the divergent shape was selected during the design phase and the dilution gas must be injected at high speed. Hence, internal diffuser and nitrogen conditions have been selected accordingly. In POLIRocket-V2, nitrogen is injected at sonic condition. The mass ratio $(M R)$ between the secondary flow and the ingested one and the mixing length $\left(L^{*}\right)$ have been investigated and the nominal values set as $10.02 \mathrm{~cm}$ and $7.5 \mathrm{~cm}$ respectively. The former defines the deceleration and cooling of the primary flow, while the latter represents the assumed length in which the mixing takes place.

- the divergent duct has been modeled considering constant area variation, friction effects and total enthalpy conservation. The computation of the properties across the shock wave is based on the normal shock wave theory. 
- stagnation conditions are considered in the collection chamber where the dump nozzle area is set in order to grant a collection chamber pressure of $2 \mathrm{bar}$, that locates the shock wave in the divergent channel, throughout steady-state mass and enthalpy balance. A nominal evaporated mass fraction $X_{e v}=0.5$ of tetrachloroethylene has been included, where 0 means no evaporation occurs. Great attention must be put on the final temperature of the particles, desired lower than the melting point of aluminum (even though particles are expected to be made of alumina only).

The quality of the code was assessed by comparing the quasi-1D method to a CFD verification campaign with the DLR TAU solver, performed by ESA, with the hybrid structured/unstructured DLR-Navier-Stokes CFD solver TAU [18]. A second order finite-volume flow solver is applied to the RANS equations while the application of the AUSMDV flux splitting scheme, in tandem with MUSCL gradient reconstruction, enables second order spatial accuracy. Turbulence modelling is implemented by means of the Spalart-Allmaras one-equation eddy viscosity model. The gas in the calculations is considered as a non-reacting, ideal gas. The initial BCs coincide with the thermo-physical properties exiting from the upstream rocket nozzle, under the assumption of no front bow shocks: nominal inlet data are listed in Table 2. Far-field conditions are set at the inlet, which is modeled with a round effects for meshing reasons. Since the axisymmetric geometry prohibits the inclusion of discrete points for nitrogen injection and dump nozzles simulation, the coolant input is realized by means of a reservoir-pressure inflow boundary condition on which the total pressure and total density of the coolant are fixed, while the collection chamber is simplified as a cylinder whose discrete outlets of the probe are neglected and the expulsion of gas is facilitated via a constant predefined pressure outlet boundary condition. For further details refer to [17].

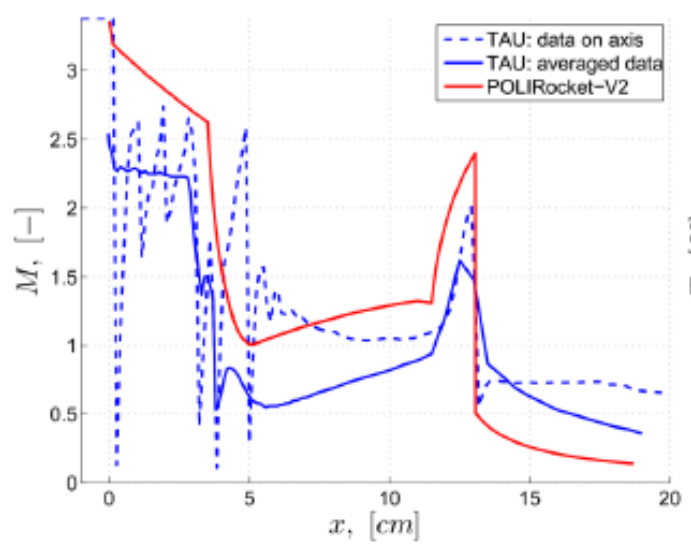

(a)

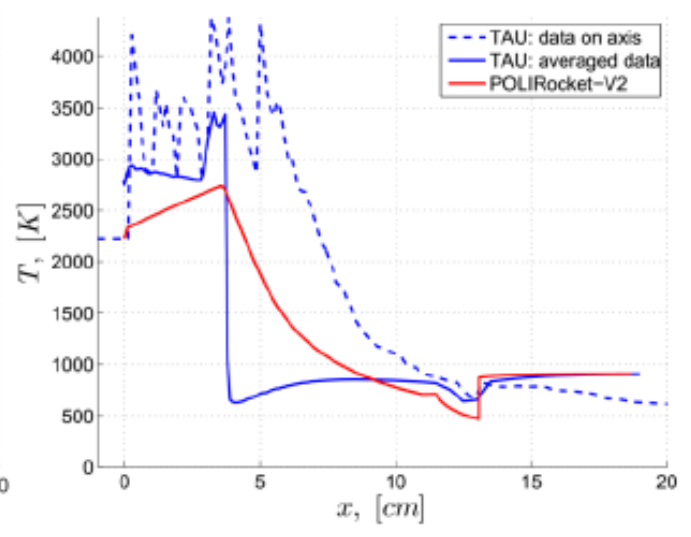

(b)

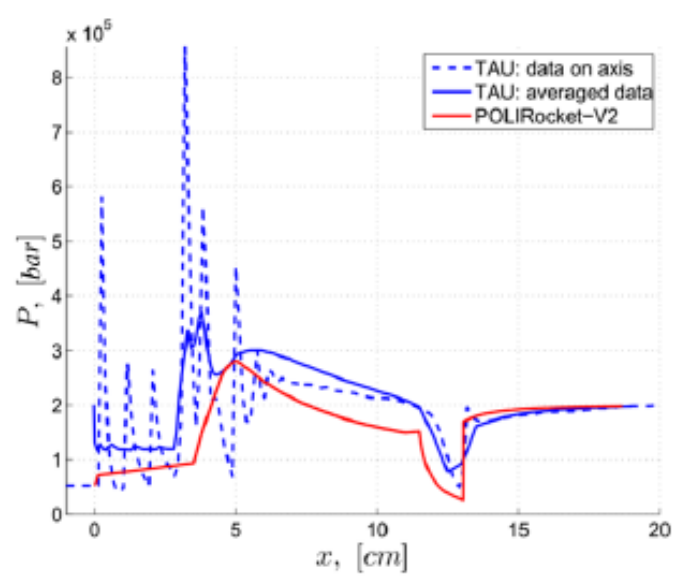

(c) 
Fig. 2 Comparison between the POLI-RocketV2 code and the TAU-DLR code: (a) Mach number, (b) static temperature and (c) static pressure.

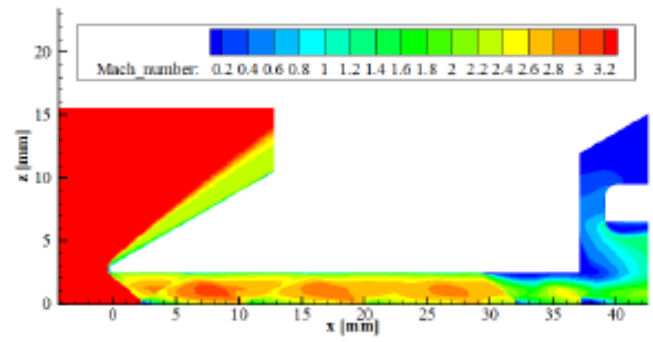

(a)

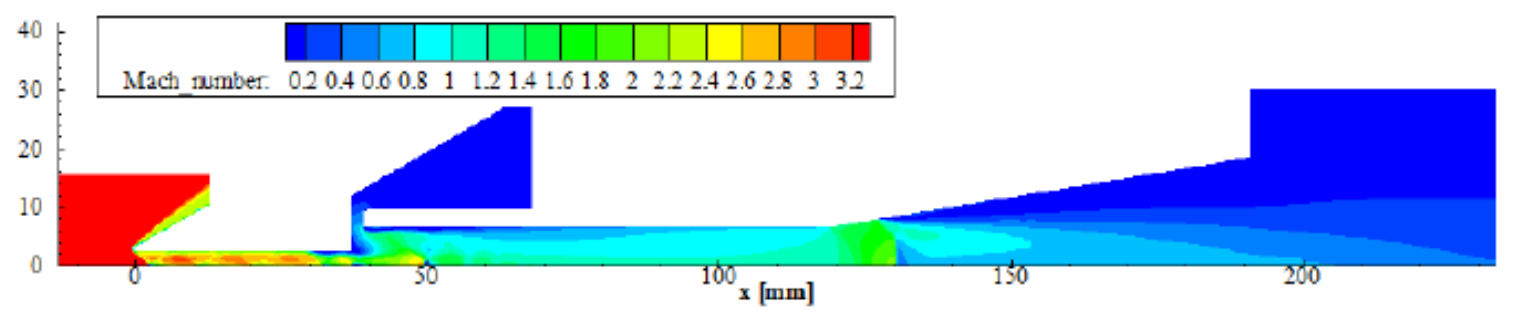

(b)

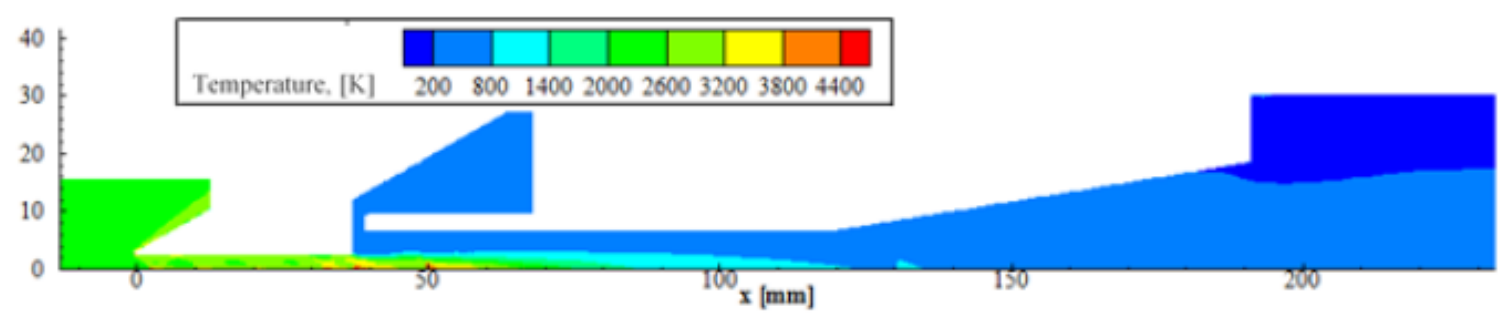

(c)

Fig. 3 Flow field within the probe: (a) magnification of the Mach number in the inlet duct; (b) Mach number; (c) static temperature.

The comparison in terms of Mach number, static temperature and pressure is highlighted in Fig. 2, while the flow evolution resulting from the CFD computation is shown in Fig. 3. Data from sampling points located on the rotational axis at one-millimeter intervals and data averaged over the channel cross section are shown. Comments on the result follow:

- according to the POLIRocket-V2 code, in the first part a Fanno flow is present, and hence a decrement of the supersonic Mach number is expected, with a consequent general increment of the static properties. A temperature enhancement greater than $2300 \mathrm{~K}$ is shown, which would cause alumina particles to melt. However, considering the assumption of adiabatic flow, it is reasonable to assume that the temperatures will be lower since in the reality a heat removal occurs due to the thermal inertia of the tip material. Moreover, the heat exchange between the plume and the external ambient would lead to an incoming colder flow with respect to the one considered in the simulations. The TAU code identifies strong fluctuations along the inlet duct, because of the oblique shock train highlighted in Fig. 3(a), dictating the different final values of the properties. The initial difference in the Mach number is due to the roundness effect of the TAU-inlet duct, which creates a short convergent channel;

- in the second portion, a progressive mass addition with corresponding increment of the main flow area defines the mixing. As far as the POLIRocket-V2 is concerned, the Mach number initially strongly decreases because

6

American Institute of Aeronautics and Astronautics 
of the momentum balance, then, the mass injection and the temperature reduction lead to a further slight increase. The same interpretation can be followed for the static pressure, while the static temperature progressively decreases because of the progressive reduction of the mixture total temperature. The same trend is observed in the CFD approach, highlighting a better mixing process far away from the rotational axis (i.e., difference between the averaged and the on-axis data). The injected coolant flow impinges on the main flow creating a subsonic region for the length of the mixing duct, as shown in Fig. 3(b).

- $\mathrm{n}$ the divergent portion a shock is located after an initial expansion and consequent acceleration. The Mach number sharply decreases, after an initial increment and the static properties increase. The shock is exactly captured by the two approaches, however the CFD results show an intrinsic bi-dimensional process occurring in the divergent channel.

\section{Off-Design Analysis}

An off-design analysis of the probe was conducted to assess its behavior under non-nominal conditions. In fact, the drawbacks of dealing with a supersonic hot flow make the design and operation of the instrument quite sensitive to deviations from the nominal behavior. Hence, POLIRocket-V2 code was used for Monte Carlo evaluations about the operational envelope of the probe for both sensitivity analysis (SA) and uncertainty analysis (UA). Sensitivity analysis is the assessment of how changes in input values impact on model outputs, qualitatively and/or quantitatively, establishing a ranking of errors; uncertainty analysis aims at determining how inputs from the system and the environment are not predictable and how these uncertainties finally produce uncertainty in the outputs. Uncertainties in the flow properties, probe geometry (i.e., inlet diameter, diameter of the radial injection area of nitrogen, nozzle diameter) and amount of evaporated tetrachloroethylene are considered: refer to Table 3 and Table 4 for details.

Table 3: Aleatory uncertainties: flow main quantities, mean value and standard deviation. A $95 \%$ of confidence level is selected, while an error of $5 \%$ is settled for $k$ during both SA and UA, of $5 \%$ for the other flow properties during SA, while of 5 and $10 \%$ during UA. The number representing the variable during SA is set.

\begin{tabular}{c|cc} 
& $\boldsymbol{\mu}^{*}$ & $\boldsymbol{\sigma}$ \\
\hline $\mathbf{T},[\mathbf{K}]$ & 2227 & 56.8 \\
$\mathbf{P},[\mathbf{P a}]$ & 53000 & 1350 \\
$\mathbf{M},[-]$ & 3.23 & 0.08 \\
$\mathbf{k},[-]$ & 1.2 & 0.307 \\
\hline
\end{tabular}

Table 4: Epistemic uncertainties: geometric parameters and evaporation rate. Minimum and maximum values are listed. The number representing the variable during $\mathrm{SA}$ is set.

\begin{tabular}{c|cc} 
& Min value & Max value \\
\hline $\mathbf{D}_{\mathbf{i n}},[\mathbf{m m}]$ & 5 & 5.6 \\
$\mathbf{g a p},[\mathrm{mm}]$ & 1.8 & 2.1 \\
$\mathbf{D}_{\mathbf{n}},[\mathbf{m m}]$ & 13.68 & 15.79 \\
$\mathbf{X}_{\mathbf{e v}}$ & 0 & 1 \\
\hline
\end{tabular}

As far as the SA is concerned, the elementary effects method (EEM) proposed by Morris has been selected for the present purpose [19], since it does not require assumption regarding the functional form of the model and it is applicable to different formulations. Moreover, it belongs to the class of the screening methods, thus aiming at identifying the subset of non-influent factors in a model using a small number of evaluations, but whose results have been demonstrated to successfully meet the performances of the widely used variance-based methods [20]. The method consists in discretizing the input space, then performing a given number of randomized on-at-time design. Each model input $X_{i}=1, \ldots, k$ is assumed to vary across $p$ selected levels in the space of the input factors. The region of experimentation is a $k$-dimensional $p$-level grid. Different elementary effects on input for each factor are estimated and averaged to obtain a global measure. Hence, each of the $r$ trajectories provides $k$ elementary effects, one per input factor.

The elementary effect $E_{i}$ is defined as:

$$
E_{i}=\frac{y\left(X_{i}, \ldots, X_{i-1}, X_{i}+\Delta, X_{i+1}, \ldots, X_{k}\right)-y(X)}{\Delta}
$$


where $\Delta$ is the variation step. The final sensitivity measures are the mean of the distribution of the absolute values of the elementary effects $\mu^{*}$ and the standard deviation $\sigma$ of these effects. The present work considers the trajectorybased sampling with a symmetric treatment of inputs proposed by Morris, with ten trajectories and seven levels [19]. The normalized results in terms of absolute mean values and standard deviations for the elementary effects related to shock wave, final pressure and final temperature are listed in Table 5. The $\mu_{j}^{*}$ is a measure of influence of the $\mathrm{j}$-th input on the output. The larger the mean is, the more the $\mathrm{j}$-th input contributes to the dispersion of the output. The $\sigma_{j}$ is a measure of non-linear and/or interaction effects of the $\mathrm{j}$-th input. If it is small, elementary effects have low variations on the support of the input. On the other hand, the larger it is, the less likely the linearity hypothesis is. Thus, a variable with a large $\sigma_{j}$ will be considered having non-linear effects or being implied in an interaction with at least one other variable. The present work will show an analysis of the impact on the shock wave position. For the tank pressure and the final temperature results listed in Table 5, same considerations would occur. Mach number, specific heat ratio and the diameter of nitrogen injection area show both a low mean value and a low standard deviation with respect to the other parameters, thus suggesting that both the contributions to the dispersion of the output and interaction/non-linear effects are not present. An interesting result is the strong influence of the temperature, pressure, inlet diameter, nozzle diameter and evaporation rate which however are characterized by very different standard deviation. In particular, analyzing the effect of the single input on the shock wave position, it was evident that the inlet diameter has a strong interaction with the other inputs, while the evaporation rate exhibits a high non-linearity effect. Three performance indexes were considered: the sum of the elementary effects of the position of the shock wave and the final temperature, the sum of the position of the shock wave and the tank pressure, and the sum of all the three single indexes. In particular, the absolute values of the normalized elementary effects have been considered to avoid negative and positive effects to cancel out, and hence to consider the net importance of the input on the output. The choice aims at underlining the importance of the location of the shock wave for the correct functioning of the probe: the final ranking of importance is listed in Table 6, where the most important parameter has the 1-st rank, while rank of two parameters having the same sum of single indexes-ranks was computed as the average of their actual final places. Moreover, both absolute (ABS) and not-absolute (N-ABS) values of the performance indexes were considered in order to highlight possible effects of negative and positive elementary effects canceling out each other. Globally the two analyses agree in the definition of the most important parameters (i.e., the inlet diameter dictating the ingested flow) and the less relevant (i.e., the specific ratio). On the contrary, as far as the intermediate positions of the final ranking are concerned, flatten contribution are highlighted in the ranking concerning not-absolute value of the elementary effects, and an overall disagreement is expected. In fact, strong interactions/non-linearity is suggested by the sensitivity analysis, as well as counter effects between parameters. Hence, all the parameters will be taken into account for the UA except for the specific heat ratio.

Table 5: The normalized results in terms of absolute mean values and standard deviations for the elementary effects related to shock wave position, tank pressure and final temperature.

\begin{tabular}{c|cc|cc|cc} 
& \multicolumn{2}{|c|}{$\boldsymbol{I}_{\boldsymbol{s} \boldsymbol{w}}$} & \multicolumn{2}{c|}{$\boldsymbol{I}_{\boldsymbol{P t}}$} & \multicolumn{2}{c}{$\boldsymbol{I}_{\boldsymbol{T f}}$} \\
\hline & $\boldsymbol{\mu}^{*}$ & $\boldsymbol{\sigma}$ & $\boldsymbol{\mu}^{*}$ & $\boldsymbol{\sigma}$ & $\boldsymbol{\mu}^{*}$ & $\boldsymbol{\sigma}$ \\
\hline $\mathbf{T}$ & 0.2297 & 0.1711 & 0.1267 & 0.2290 & 0.4475 & 0.6812 \\
$\mathbf{P}$ & 0.2042 & 0.1007 & 0.0000 & 0.0000 & 0.0015 & 0.0022 \\
$\mathbf{M}$ & 0.0151 & 0.0324 & 0.1574 & 0.3648 & 0.5288 & 0.6764 \\
$\mathbf{k}$ & 0.0233 & 0.0095 & 0.0555 & 0.0912 & 0.1140 & 0.1885 \\
$\mathbf{D}_{\text {in }}$ & 0.3193 & 0.9386 & 0.2220 & 0.1422 & 0.6441 & 0.1778 \\
$\mathbf{g a p}$ & 0.0249 & 0.0495 & 0.1155 & 0.1411 & 0.3036 & 0.1062 \\
$\mathbf{D}_{\mathbf{n}}$ & 0.4169 & 0.0747 & 0.4444 & 0.3456 & 0.0031 & 0.0041 \\
$\mathbf{X}_{\mathbf{e v}}$ & 0.7927 & 0.2654 & 0.8343 & 0.8041 & 0.0057 & 0.0079 \\
\hline
\end{tabular}

8

American Institute of Aeronautics and Astronautics 
Table 6: Real and absolute normalized values for performance indexes and corresponding final ranking.

\begin{tabular}{c|c|c|c|c} 
Ranking & ABS TOT & $\begin{array}{c}\text { ABS Final } \\
\text { Ranking }\end{array}$ & $\begin{array}{c}\text { N-ABS } \\
\text { TOT }\end{array}$ & $\begin{array}{c}\text { N-ABS Final } \\
\text { Ranking }\end{array}$ \\
\hline $\mathbf{T}$ & 11 & 4 & 14 & 4.5 \\
$\mathbf{P}$ & 17 & 6 & 13 & 3 \\
$\mathbf{M}$ & 16 & 5 & 8 & 2 \\
$\mathbf{k}$ & 24 & 8 & 22 & 8 \\
$\mathbf{D}_{\text {in }}$ & 6 & 2 & 3 & 1 \\
$\mathbf{g a p}$ & 20 & 7 & 14 & 4.5 \\
$\mathbf{D}_{\mathbf{n}}$ & 10 & 3 & 17 & 6.5 \\
$\mathbf{X}_{\mathbf{e v}}$ & 4 & 1 & 17 & 6.5 \\
\hline
\end{tabular}

As far as the uncertainty analysis is concerned, the input errors propagation is studied as in the framework proposed by Oberkampf et al. in [21], hence considering a double loop or nested sampling for the mixed aleatory-epistemic uncertainties investigation. Each of the samples obtained from an aleatory uncertainty is associated with a probability of occurrence. For each sample of epistemic uncertainties, the aleatory uncertainties are propagated through the model throughout out Latin hypercube sampling to produce a single CDF, being the epistemic parameter simply a possible realization over an interval-valued range of the input quantity. Values considered are listed in Table 3 and Table 4. The final result is an ensemble of CDFs, whose widest extent form a probability box (p-box). A p-box shows an interval-valued probability. Since the key point of the design is the supersonic-to-subsonic transition inside the divergent channel, the collection chamber and the shock wave position in the divergent must be monitored. Hence, UA enables to detect and quantify failures:

- failure 1: it is related to the reaching of the choking condition in the mixing chamber, an unintended situation, since it is both not supported by the present code and not stable from the fluid dynamics view point;

- failure 2: it is due to a low collection chamber pressure that is not able to set a shock wave in the divergent;

- failure 3: it is related to a too high tank pressure and a shock wave pushed in the mixing chamber.

In case of any failure occurrence, the problematic sample is discharged, and it is not considered in the statistical analysis. Results of UA allow to make the following considerations:

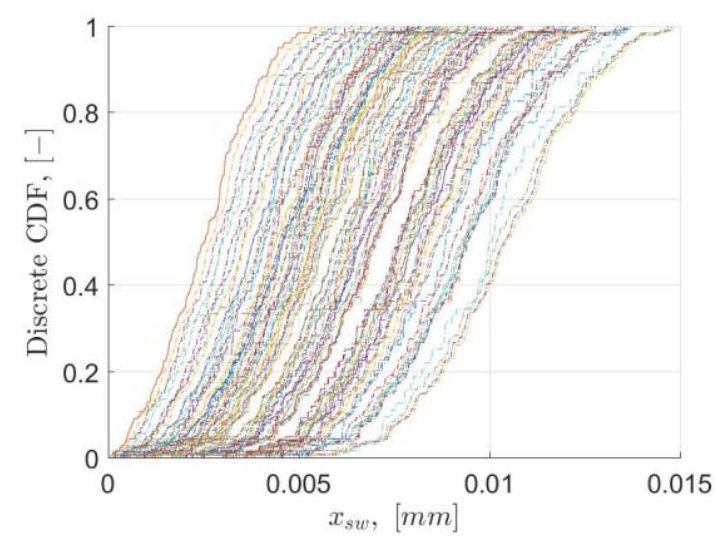

(a)

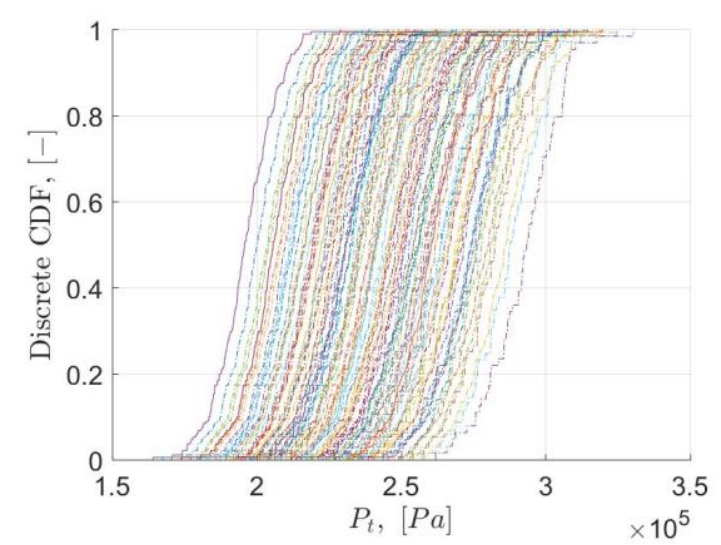

(b)

Fig. 4 P-box distribution of (a) shock wave position and (b) tank pressure for the UA with an error on the aleatory uncertainties of $5 \%$.

- Concurrent effects in the definition of the shock wave position, leading to a symmetrical distribution around the nominal value (i.e., $0.008 \mathrm{~mm}$ far from the inlet section of the divergent), while the uncertainties on tank pressure are mostly directed towards its increase (i.e., nominal value of 2 bar), are shown by Fig. 1. Analysis on the impact of the inputs increased on at time leads to the same consideration. In fact, the shock wave is pushed towards the end of the divergent by an increment of $\mathrm{P}, \mathrm{M}, \mathrm{k}$ and nozzle diameter, towards the inlet of the divergent by an increase of $\mathrm{T}$ and nitrogen injection diameter, while the evaporation rate and the inlet diameter show non-linear effects. On the contrary, most of the input parameters dictates an increase in the pressure tank in a more marked way; 
- when considering 5\% of errors in the flow properties, failures are essentially caused by the chocking of the mixing chamber (9.6\%). This type of failure is not related to the amount of evaporated tetrachloroethylene. On the contrary, it plays a role in the failures occurrences due to high pressure in the collection chamber pushing the shock wave toward the mixing chamber (Failure 3). The percentage of Failure 3 is null for 5\% errors and extremely low (1\%) and limited to the total evaporation of the quenching liquid for $10 \%$ errors, which cause $10.1 \%$ of failures due to the chocking. Failures 2 does not occur.

\section{Experimental Campaign}

The proof of concept of the fluid dynamic model and of the collection methodology was achieved throughout a cold flow experimental campaign at a representative Mach number (i.e., $M=3$ ). The tests were performed at the vertical wind tunnel facility (VMK) of DLR (Cologne) [22].
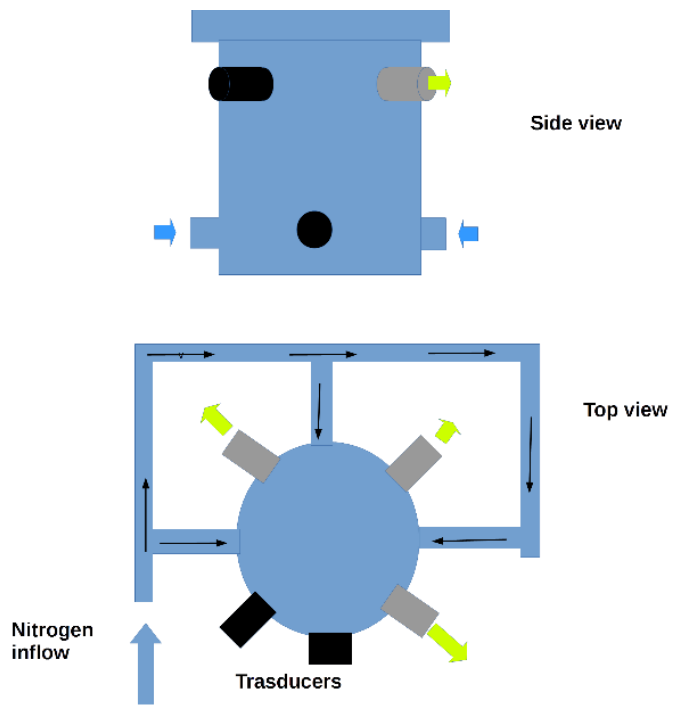

(a)
Fig. 5 Experimental setup: (a) schematic representation of the nitrogen supply; (b) interface Probe-VMK: probe connected to a supporting arm.

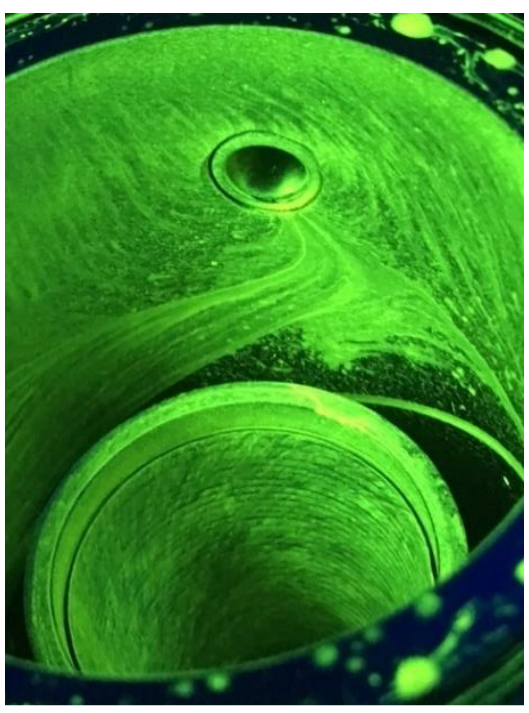

Fig. 6 Oil flow test: swirl flow pattern.

The VMK operates with a contour nozzle granting a Mach number equal to 3 at the exit section and expanding a flow at ambient temperature and at different total pressures. In addition to high speed Schlieren visualization devices 
operating during all the cold flow tests, in-house seeding generator for dispersing solid particles is implemented for the testing of particles-collection methodology. Water has been used in place of tetrachloroethylene to simulate the quenching liquid, while as shown in Fig. 5(a), a non symmetrical injection of nitrogen has been implemented, giving rise to a spinning flow inside the probe that could enhance the mixing with the ingested mass flow rate. Fig. 6 features the flow pattern obtained by an oil flow test. Possible criticality of this solution could be the depletion of part of the collection liquid (i.e., quenching liquid and solid particles) due to the spinning flow, which would be overcome inserting in the collection chamber a vortex breaker. The required nitrogen mass flow rate is controlled by a Bronkhorst flowmeter (Bronkhorst IN-Flow F-116BI-IIU-90-V), while a relay switch circuit controlled by Arduino dictates the opening and closure of an electrovalve which assures the correct functioning of the injector for 1 second. Off-design tests at different channel total pressures and hence mass ratio between the ingested flow and the nitrogen supply, whose mass was fixed to $0.042 \mathrm{~kg} / \mathrm{s}$, were performed.

The fluid dynamic behavior during the transient start up and steady state conditions at 15 bar can be observed in Fig. 7. Schlieren visualizations enable to infer the bow shock in front of the inlet during the start-up phase and the attached shock wave and the Mach disk, suggesting the correct functioning and hence the robustness of the system during off-design testing. In fact, the rocket exhaust plume consists of two different parts: an approximately inviscid non-reacting core and a surrounding viscous mantle wherein reactions take place by mixing with the atmosphere. If the flow is slightly under-expanded, it keeps expanding outside the nozzle through Prandtl-Meyer fans until the dynamic pressure of the external flow forces it to turn. Considering a supersonic exhaust plume, oblique shock waves only enable this mechanism to occur, propagating to the plume axis where they are reflected. When the flow crosses the discontinuity, a compression occurs, achieving a pressure value higher than the ambient one. Hence, an expansion fan occurs. The process repeats itself forming a pattern of Mach diamonds. If the ratio of exit to ambient pressure is high, the plume expansion increases accordingly with stronger shocks. When momentum and energy of the flow cannot remain conserved in an inviscid flow across an oblique shock as it approaches the plume axis, a normal shock wave called Mach disk is formed. Downstream of it, the flow is subsonic. For an extensive discussion of the plume phenomenology, refer to Simmons et al. [23]. Hence, a supersonic ingestion is demonstrated.

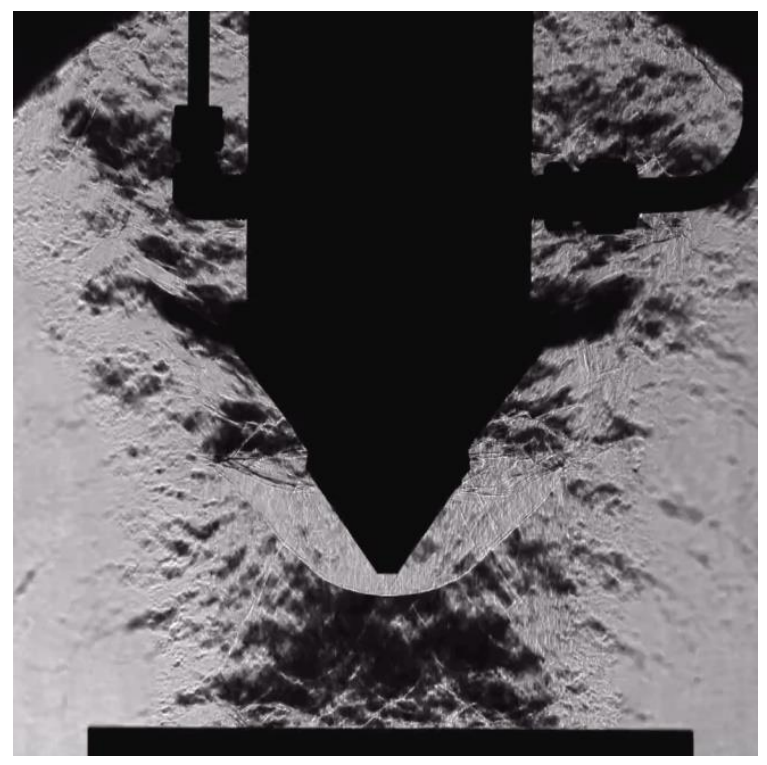

(a)

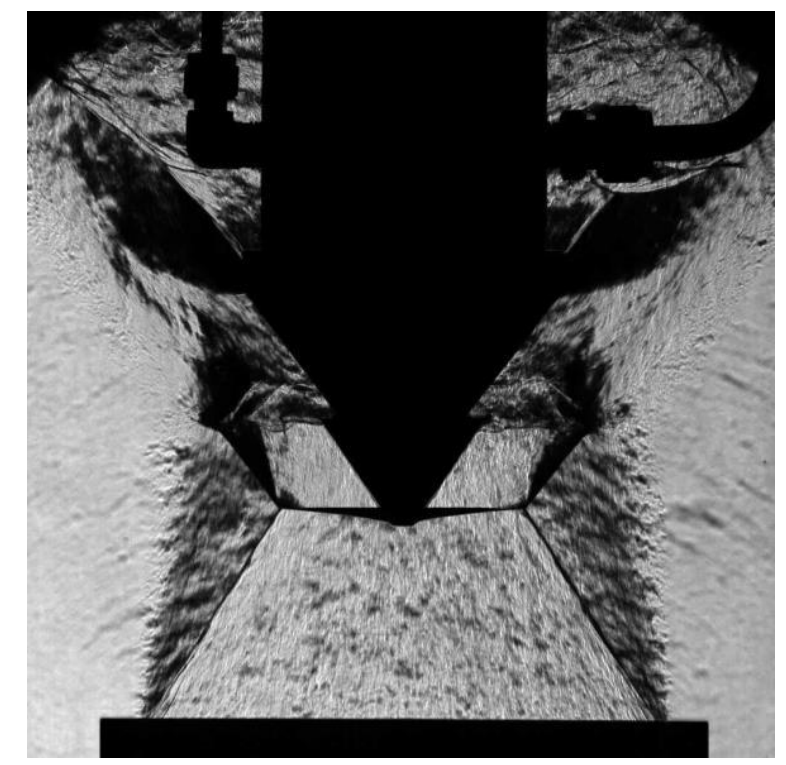

(b)

Fig. 7 Cold flow test at 15 bar: (a) bow shock appears during the transient start up; (b) steady state condition.

Table 7. Comparison of relevant diameters between the original and the collected particles.

\begin{tabular}{l|cc} 
& $\mathbf{D}(\mathbf{3}, \mathbf{2}),[\boldsymbol{\mu m}]$ & $\mathbf{D}(\mathbf{4}, \mathbf{3}),[\boldsymbol{\mu} \mathbf{m}]$ \\
\hline Original & 5,693 & 17,032 \\
Collected & 5,579 & 16,993 \\
\hline
\end{tabular}


The collection method validity was verified during seeding test featuring micrometric magnesium oxide. The particle size distribution was obtained using laser diffraction methodology with Malvern Mastersizer 2000 instrument with water dispersion. Results in terms of $\mathrm{D}(4,3)$ and $\mathrm{D}(3,2)$ greatly fit the original size distribution mean diameter, suggesting an overall equality between the two batches of particles, as shown in Table 7 for tests at 25 bar.

\section{Conclusion}

A supersonic probe for particles collection has been designed in the EMAP framework. It swallows a supersonic flow which is cooled and decelerated till reaching subsonic conditions by a controlled mixing with a secondary flow. The particles are then quenched and collected for post-tests analysis. The design was joined by quasi 1D gas dynamics and normal shock wave theory. The code developed was based on Shapiro equations and was verified against an asymmetric CFD simulation performed with the DLR-TAU code. The location of normal shock wave was found to be properly captured, as well as the variables trend. Intrinsically more-than-1D processes could not be identified, hence pressure and temperature trends computed with POLI-RocketV2 appeared to be smoother than in the CFD results. However, the execution time of the proposed quasi-1D approach is orders of magnitude shorter than a CFD solution, proposing itself as a reliable smart engineering approach featured by low computational demands. The robustness of the system was tested throughout a sensitivity and uncertainty analysis, as well as off-design cold flow tests. In particular, concurrent effects in the definition of the shock wave position were highlighted leading to a 10\% of failure of the system due to chocking reached in the mixing chamber when considering a $10 \%$ of errors of the ingested flow properties. The proof of concept of the method has been obtained during cold flow tests at relevant Mach number (i.e., $M=3$ ). Supersonic ingestion occurred, and particles were collected and analyzed with laser granulometry diffraction.

\section{Acknowledgments}

This activity was supported by the EMAP (Experimental Modeling of Alumina Particulate in Solid Booster) project, an ESA-funded research activity (ESA contract No. 4000114698/15/NL/SFe).

\section{References}

[1] Voigt C., Schumann, U., Graf, K. and Gottschaldt, K. D., "Impact of rocket exhaust plumes on atmospheric composition and climate-an overview", EUCASS Proceedings Series-Advances in Aerospace Sciences, Vol. 4, 2013, pp. 657-670.

[2] Hermsen, R., “Aluminum Oxide Particle Size for Solid Rocket Motor Performance Prediction”, Journal of Spacecraft and Rockets, Vol. 18, No. 6, 1981, pp. 483-490.

[3] Sambamurthi, J., "Al2O3 collection and sizing from solid rocket motor plumes", Journal of Propulsion and Power, Vol. 12, No. 3, 1996, pp. 598-604.

[4] Koch, A., Bauer, C., Dumont E., Minutolo, F., Sippel, M., Grenard, P., Ordonneau, G., Winkler, H., Guénot, L., Linck, C., Wood, C., Vira, J., Sofiev, M., and Tarvainen, V., "Multidisciplinary approach for assessing the atmospheric impact of launchers", $4^{\text {th }}$ CEAS Air \& Space Conference, Linköping University Electronic Press, 2013, pp. 340-346.

[5] Sullivan, R., Thornberry, T., and Abbatt, J., "Ozone decomposition kinetics on alumina: effects of ozone partial pressure, relative humidity and repeated oxidation cycles", Atmospheric Chemistry and Physics, Vol. 4, No. 5, 2004, pp. 1301-1310.

[6] Bianco, R., and Hynes, J., "Heterogeneous Reactions Important in Atmospheric Ozone Depletion: A Theoretical Perspective", Vol. 39, No. 2, 2006, pp. 159-165.

[7] Sullivan, R., Thornberry, T., and Abbatt, J., "Ozone decomposition kinetics on alumina: effects of ozone partial pressure, relative humidity and state of film oxidation”, Atmospheric Chemistry and Physics Discussions, Vol. 4, No. 2, 2004, pp. 1977-2002.

[8] Brown, B. and McArty, K.P., "Particle size of condensed oxides from combustion of metalized solid propellants", Symposium (International) on Combustion, Vol. 8, 1961, pp. 814-823.

[9] Cheung, H., and Cohen, N. S., "Performance of solid propellants containing metal additives", AIAA Journal, Vol. 3, No. 2, pp. 250-257, 1965

[10] Sehgal, R. “An experimental investigation of a gas-particle system”, DTIC Document, Tech. Rep., 1962.

[11] Dobbins, R. A., and Strand, L. D., "A comparison of two methods of measuring particle size of al2o3 produced by a small rocket motor”, AIAA Journal, Vol. 8, No. 9, pp. 1544-1550, 1970.

[12] Carns, R. H., Armstrong, G., Rast, R. H., Mitchell, D. R., Washington, DC, US Patent No. 6,964,69, filed 15 Nov. 2005 . 
[13] Kessel, P. A., Washington, DC, US Patent No. 4,622,216, filed 5 May 1987.

[14] Carlotti, S., Ferreri, A., Bisin, R., Maggi, F., Galfetti, L., Saile, D., Gülhan, A., and Langener, T., "Development of a Probe for Particle Collection in High-Temperature, Supersonic Flow: Conceptual and Detailed Design", 7th European Conference for Aerospace Sciences, 2017.

[15] Babuk, V., Vasilyev, V., Malakhov, M.,"Condensed combustion products at the burning surface of aluminized solid propellant", Journal of Propulsion and Power, Vol 15, No. 6, 1999, pp 783-793.

[16] Gallier, S., Kratz, J.-G., Quaglia, N., Fouin, G., "Detailed analysis of a quench bomb for the study of aluminum agglomeration in solid propellants", Progress in Propulsion Physics, Vol 8, 2016, pp 197-212.

[17] Maggi, F., Ferreri, A., Bisin, R., Carlotti, S., Galfetti, L., Groll, C., and Langener, T., Development of a Probe for Particle Collection in High-Temperature, Supersonic Flow: Application of Quasi-1D Engineering and 2D Axisymmetric CFD”, 7th European Conference for Aerospace Sciences, 2017.

[18] Anon, Homepage of dlr tau code, last Visit: 13/01/2018. URL http://tau.dlr.de/code-description/

[19] Morris, M.D., "Factorial sampling plans for preliminary computational experiments. Technometrics, Vol. 33, No. 2, pp. 161-174, 1991.

[20] Campolongo, F., and Saltelli, A., "Sensitivity analysis of an environmental model: an application of different analysis methods". Reliability Engineering \& System Safety, Vol. 57, No. 1, pp. 49-69, 1997.

[21] Oberkampf, W. L., DeLand, S. M., Rutherford, B. M., Diegert, K. V., and Alvin K. F. "Error and uncertainty in modeling and simulation”. Reliability Engineering \& System Safety,Vol. 75(3), pp 333-357, 2002.

[22] Vertical test section cologne (VMK), supersonic and hypersonic technology department. URL http://www.dlr.de/as/en/desktopdefault.aspx/tabid-194/407_read-5445/

[23] Simmons, F.S., "Rocket exhaust plume phenomenology", Aerospace Corporation, 2000. 